\title{
Noncritical Liouville string escapes constraints on generic models of quantum gravity
}

\author{
John Ellis \\ Theoretical Physics Division, CERN, CH-1211 Geneva 23, Switzerland
}

N. E. Mavromatos

Theoretical Physics Group, Department of Physics, King's College London, Strand, London WC2R 2LS, United Kingdom

D. V. Nanopoulos

Department of Physics, Texas A \& M University, College Station, Texas 77843, Astroparticle Physics Group, Houston Advanced Research Center (HARC), Mitchell Campus, Woodlands, Texas 77381, and Academy of Athens, Division of Natural Sciences, 28 Panepistimiou Avenue, Athens 10679, Greece

(Received 21 August 2001; published 8 February 2002)

\begin{abstract}
It has recently been pointed out that generic models of quantum gravity must contend with severe phenomenological constraints imposed by gravitational Čerenkov radiation, neutrino oscillations and the cosmic microwave background radiation. We show how the noncritical Liouville-string model of quantum gravity we have proposed escapes these constraints. It gives energetic particles subluminal velocities, obviating the danger of gravitational Čerenkov radiation. The effect on neutrino propagation is naturally flavor independent, obviating any impact on oscillation phenomenology. Deviations from the expected blackbody spectrum and the effects of time delays and stochastic fluctuations in the propagation of cosmic microwave background photons are negligible, as are their effects on observable spectral lines from high-redshift astrophysical objects.
\end{abstract}

DOI: 10.1103/PhysRevD.65.064007

PACS number(s): 04.60.- m, 11.25.Pm

\section{INTRODUCTION}

A testable quantum "theory of everything," including gravity, is the analogue for particle physicists of the "faint blue dot" towards which much space research is directed. One might seek to test quantum theories of gravity either through subtle effects in a conventional physical framework, such as non-renormalizable operators scaled by some inverse power of the Planck mass $m_{P}$, or through some qualitatively new effect, such as a violation of quantum mechanics and/or relativity. The latter is a rather dangerous route for models of quantum gravity to follow, since both quantum mechanics and relativity are known to be very robust theories that are difficult to make "slightly pregnant": any deviation from their sacred canons risks catastrophic phenomenological consequences.

We have proposed a formulation of quantum gravity based on noncritical string theory $[1,2]$ with a Liouville field playing the role of time [3]. This model suggests that there might be observable violations of quantum mechanics, and that energetic particles might travel at less than the velocity of (low-frequency) light. Liouville string theory passes many non-trivial consistency tests, e.g., of the interpretation of the Liouville field as time [3], the appearance of an eleventh dimension in $M$ theory [4], and the Helmholtz conditions for quantization [5] in theory space.

Several accelerator and non-accelerator experiments are already sensitive to violations of quantum mechanics and/or relativity that challenge candidate models of quantum gravity. For example, probes of quantum mechanics in the $K^{0}-\bar{K}^{0}$ system $[6,7]^{1}$ are sensitive to violations of quantum mechanics that are scaled by one inverse power of $m_{P}$ [9], and probes of light propagation from distant astrophysical sources are sensitive to deviations from special relativity that are also scaled by one inverse power of $m_{P}$. The latter might also play a role in the possible evasion [10] of the GreisenZatsepin-Kuzmin cutoff [11] by ultrahigh-energy cosmic rays (UHECR) [12] and energetic astrophysical photons.

Recently, several potential pitfalls for candidate quantum theories of gravity have been pointed out. In theories in which energetic particles travel faster than low-energy gravitons, UHECR emit gravitational Čerenkov radiation at catastrophic rates, causing them to lose energy and become unobservable [13]. Neutrino-oscillation phenomenology would be drastically modified in candidate quantum theories of gravity that have flavor-dependent effects on neutrino propagation [14]. Most recently, it has been argued that the lightcone fluctuations expected in generic quantum theories of gravity have problematic consequences for the cosmic microwave background radiation [15].

It is the point of the present note to demonstrate how noncritical Liouville string escapes these potentially fatal constraints.

In this approach, UHECR automatically travel subluminally, so the issue of gravitational Cerenkov radiation does not arise. The quantum-gravitational effects on neutrino propagation are naturally flavor independent, so that effects

\footnotetext{
${ }^{1}$ See also [8] for a discussion of similar effects in the $B^{0}-\bar{B}^{0}$ system.
} 
on neutrino-oscillation phenomenology are negligible. Finally, the effects on the blackbody spectrum of the cosmic microwave background radiation, including those due to the expected delays and fluctuations in the propagation of microwave background photons, are also negligible, as are their expected effects on the spectral lines of astrophysical sources at high redshifts.

\section{GRAVITATIONAL ČERENKOV RADIATION AND NONCRITICAL STRING THEORY}

We recall that an important constraint on some models of quantum gravity is imposed by the absence of gravitational Cerenkov radiation (GCR). This was recently pointed out in [13], in the context of a $D$-brane scenario of warped gravity [16], but the remark is more general.

In the warped-gravity framework, our four-dimensional world is given a dual interpretation [17] as a warped spacetime [16], associated with a D3-brane embedded in a higherdimensional (bulk) geometry. The purely four-dimensional interpretation of such geometries is due to the AdS/ conformal field theory (CFT) correspondence [18]. The ensuing violation of four-dimensional Poincaré invariance implies that gravitational waves on our brane world always travel with a speed $c_{G}$ lower than the speed of light $c$. The fact that gravity propagates at a speed less than that of light implies the appearance of GCR in such models [13], in analogy with the conventional electromagnetic Cerenkov radiation (ECR) emitted by any charged particle propagating in a medium where the velocity of light is less than that of the particle, which may occur when the medium's refractive index $n>1$. ECR is emitted because the charged particle outruns its own electromagnetic field in the medium, propagating "superluminally." In a similar spirit, if gravitational waves propagate slower than light or ultrarelativistic particles in our brane world, GCR pheomenon will occur, even if the particle is uncharged, since all particles couple to gravity.

Based on this observation, the observed UHECR were used to derive restrictions [13] on the deviation of the "gravitational refractive index" $n_{G}$ from 1 , placing a lower bound on the propagation speed of gravity in such warped space-time scenarios. Simple kinematics fix the rate of loss of energy due to GCR, which may be used to estimate the maximum total time of travel possible for the observed UHECR [13]:

$$
t_{\max }=M_{P}^{2} /\left(n_{G}-1\right)^{2} p^{3}
$$

where $M_{P}$ is the Planck mass, and $n_{G}>1$ is the gravitational refractive index. The observed UHECR with energies higher that $10^{11} \mathrm{GeV}$ require $n_{G}-1<10^{-15}$, and an even more stringent bound: $n_{G}-1<10^{-19}$ can be derived in some models for the production of UHECR. These limits may be problematic for some warped-space-time models.

This argument can also be used to constrain models in which low-energy gravitons, photons and other particles travel at speeds close to the velocity of light, so that effectively $n, n_{G} \approx 1$ at low frequencies, whereas ultrahigh-energy particles travel faster than the speed of light: $n_{U H E}<1$. Specifically, an analysis similar to that of [13] shows that one also has

$$
t_{\max }=M_{P}^{2} /\left(1-n_{U H E}\right)^{2} p^{3}
$$

An example of such a model would be one in which energetic particles travel at velocities that differ from the speed of light by amounts that increase with energy:

$$
v(E)=c\left(1+\xi\left(\frac{E}{M_{Q G}}\right)^{p}+\cdots\right)
$$

with $^{2} \xi=+1$ and $p>0$, resulting in $1-n_{U H E} \simeq\left(E / m_{Q G}\right)^{p}$. In this case, the bound of [13] may be translated into

$$
m_{Q G}>10^{11+15 / p} \mathrm{GeV}
$$

which might be problematic for models with $m_{Q G}$ $\sim 10^{19} \mathrm{GeV}$ and $p=1$, in particular.

In our noncritical Liouville model for quantum gravity, we have predicted that the velocities of energetic particles should deviate from the speed of light in a manner similar to Eq. (3) with $p=1$. However, in our model, we have also predicted that $\xi=-1$, i.e., energetic particles travel at less than the speed of light. Hence the constraint (4) does not apply. We now give an intuitive explanation of the sign of $\xi$, and then recall the formal derivation that $\xi=-1$.

We envisage that, in any quantum theory of gravity, space-time will no longer be (approximately) flat at short distances, since quantum fluctuations in the background metric will endow it with a "foamy" structure. This will give rise to microscopic "gravitational lensing" phenomena, in particular time delays compared to propagation in a flat space-time. These we expect to be relatively unimportant for long-wavelength probes that average out many microscopic fluctuations, whereas very-short-wavelength probes would be systematically delayed, as they "bump over" all the corrugations in space-time. This rough and intuitive picture suggests that $\xi$ should be negative and $p>0$, as we find in detailed calculations in our noncritical Liouville model for quantum gravity [19] that we now review.

In this approach, one may view our world as a three-brane punctured by $D$-particle defects that deform space-time in their neighborhoods when struck by an energetic particle or string state. The presence of recoiling defects in our brane world breaks explicitly Poincaré invariance, and Lorentz invariance in particular. We have dealt specifically with scalar closed-string modes for illustrative purposes. However, the results can easily be extended to particles of non-zero spin. In particular, we have derived [20,21] a Born-Infeld modification of Maxwell electrodynamics, and shown that photons propagate at less than the speed of light in the corresponding modifications of Maxwell's equations. We have also demon-

\footnotetext{
${ }^{2}$ We may always absorb the magnitude of $\xi$ into that of the quantum-gravity scale $m_{Q G}$, so that the two distinguishable cases are $\xi= \pm 1$.
} 
strated a corresponding modification of the speeds of energetic fermions [22], to which we return anon.

The basic idea is that, when an energetic string state strikes a $D$-particle defect in our four-dimensional brane world, the latter recoils at the moment of impulse, and hence the space-time surrounding the defect is distorted. Formally, the distortion of space-time is described by a deviation from the conformal symmetry of the string theory background, which is restored by Liouville dressing, with the Liouville mode identified as target time [3]. With such a dressing, one obtains, in the mean-field approximation, a distortion of the initially flat space-time characterized by the following nonzero off-diagonal element in the metric tensor:

$$
G_{0 i}=u_{i} \Theta(t), \quad t \gg 1,
$$

where $u_{i} \sim E / m_{D}$ is the recoil velocity of the $D$ particle of mass $M_{D} \sim M_{s} / g_{s}: M_{s}=1 / l_{s}$ is the string scale, and $g_{s}$ is the string coupling. The corresponding modification of Maxwell's equations and the ensuing reduction in the velocity of light is discussed in [23].

The mean-field quantum-gravity result (5) leads, when one considers null geodesics in the background metric, to an energy-dependent refractive index $n_{Q G}(E)$ :

$$
n_{Q G}(E)=c\left(1+\xi\left(\frac{E}{M_{D}}\right)\right)
$$

where $\xi=-|\mathcal{O}(1)|<0$. The appearance of the $D$-brane scale, replacing the generic quantum-gravity scale $m_{Q G}$, is a consequence of the rôle of $D$-particle excitations as the dominant ingredients in quantum space-time foam in this model. As mentioned above, the appearance of a subluminal effective speed of light is a consequence of the Born-Infeld dynamics induced by the recoiling $D$ particles $[21,19]$.

In addition to this mean-field refractive-index effect, there are stochastic effects on particle propagation. To understand their origin, one must distinguish our foam model from a situation in which $D$-particle defects are real. In our foam model, the defects are viewed as virtual excitations or fluctuations of the quantum stringy gravity vacuum. In a naive diagrammatic sense, therefore, one may consider a selfenergy loop diagram for a propagating particle, in which a $D$-particle defect is emitted and then re-absorbed after a time interval $t_{D}^{\text {stoch }} \sim t_{P}$. The emission process causes the particle to "lose" energy to the defect, which is "regained" after a time $\sim t_{P}$ during the reabsorption. Thus, in space-time foam energy is conserved on the average for the propagation of particles. ${ }^{3}$ During the emission and re-absorption processes, there is a non-trivial gravitational distortion of space-time, which results in the formation of a "bubble" with a refractive index that fluctuates non-trivially, as discussed in [19] and reviewed briefly here.

\footnotetext{
${ }^{3}$ However, energy may not be conserved in decays [24]
}

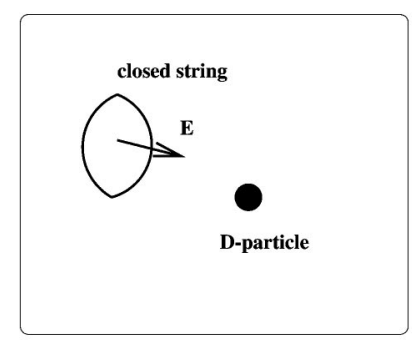

(a)

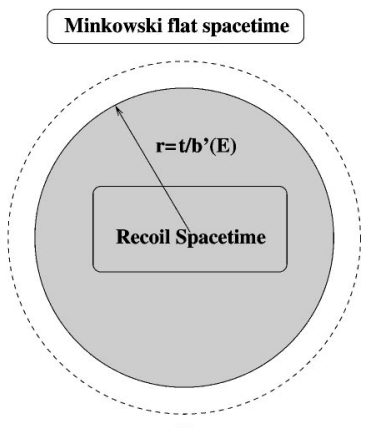

(b)
FIG. 1. (a) Scattering of a closed-string mode of energy $E$ off a $D$ particle embedded in a four-dimensional space-time. (b) After the recoil an effective bubble (shaded region) is formed, inside which particles travel at less than the (low-energy) speed of light, whereas the exterior geometry is flat Minkowski. The bubble is unstable and shrinks by evaporation, as indicated by the dashed circle.

The interaction of an energetic particle with the $D$-particle defects leads, in good approximation, to Gaussian fluctuations in the recoil velocity of the $D$ particle, with a width of order [19]:

$$
\Delta^{\text {stoch }} u_{i}=\Gamma-\Gamma(u=0)=\mathcal{O}\left(g_{s}^{2} \frac{\bar{u}^{2}}{2}\right)
$$

These uncertainties in the recoil velocity "lost" to the foam imply corresponding fluctuations in the energy $\Delta E$ of the propagating particle:

$$
\Delta^{\text {stoch }} E \sim \Delta E_{\text {kin }} \sim|\bar{u}|^{2} \frac{\Delta^{\text {stoch }} u}{u} M_{D} \sim \frac{g_{s}^{2} E^{3}}{M_{D}^{2}} .
$$

These fluctuations can be related to the resummation of world-sheet genera that corresponds to higher-order quantum effects in the $D$-particle model of space-time foam. Formally, they arise because of modular infinities associated with specific deformations of the world-sheet $\sigma$ model that have zero conformal dimension, and are related to the logarithmic operators characterizing the $D$-particle recoil [19].

One may treat such effects by regarding [19] the disturbance of space-time due to the recoil of the heavy defect as creating a microscopic space-time "bubble," as seen in Fig. 1 , whose radius is of the same order as the inverse quantum uncertainty $b^{\prime}(E)$ in the momentum of the heavy $D$ particle [21]:

$$
b^{\prime}(E)^{2}=4 g_{s}^{2} M_{s}\left(1-\frac{285}{18} g_{s}^{2} \frac{E^{2}}{M_{D}^{2}}+\cdots\right)
$$

where $E$ again denotes the energy of the incident particle. We note that $b^{\prime}(E)$ decreases with increasing energy [21]. As argued in [19], the formation of such "bubbles" induces quantum fluctuations in the two-point function for the metric (5), which in turn induce stochastic corrections in $G_{00}$ component of the metric: 


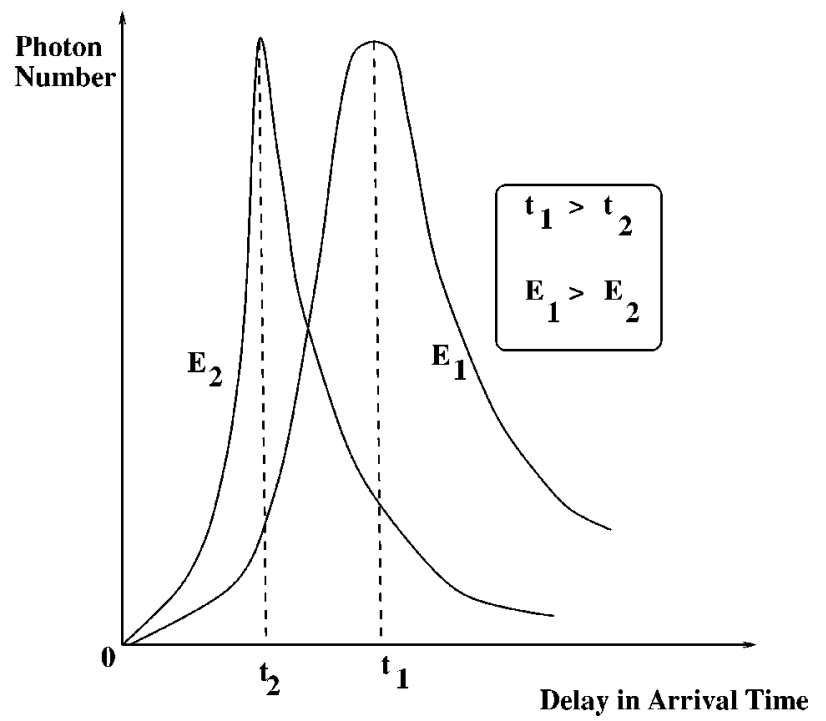

FIG. 2. Illustration of the quantum-gravity induced refractive index (6) and stochastic effect (11). The delays in the arrival times $t_{i}$ of photons increase with increasing energy $E_{i}$, due to the subluminal character of the effect (6). The stochastic effects (11), on the other hand, imply that the width of a pulse of photons with the same energy spreads out more for the more energetic channels, although the magnitude of the effect is smaller than the retardation.

$$
\Delta G_{00}=\frac{b^{\prime}(E)^{2} r^{2}}{t^{2}}: \quad r^{2}=\sum_{i=1}^{3} x_{i}^{2}
$$

where $b^{\prime}(E)$ is given by Eq. (9) when $E \ll M_{D}$.

The energy-independent part of Eq. (10) proportional to the first term in Eq. (9), i.e., proportional to $g_{s}^{2}$, should be subtracted and absorbed into the conventional Minkowski metric. The remaining E-dependent parts in Eq. (10), when computed near the light-cone $r \sim t / 2 g_{s}$ where the recoil space-time is matched with the external Minkowski spacetime [25], yield stochastic fluctuations about the previous mean-field refractive-index effect (6):

$$
\left(\Delta n_{Q G}(E)\right)_{\text {stoch }}=\mathcal{O}\left(g_{s}^{4} \frac{E^{2}}{M_{D}^{2}}\right) .
$$

We observe that this stochastic effect is suppressed relative to the mean-field refractive-index effect (6) by two powers of $g_{s}^{2}$ and of $E / M_{D} \cdot{ }^{4}$ Hence, the dominant effect in this approach will be a reduction in the resulting speed of energetic particles, with small fluctuations around the mean-field velocity, ${ }^{5}$ as seen in Fig. 2.

We conclude that particle propagation is always subluminal in this approach, a property traceable to the characteristic

\footnotetext{
${ }^{4}$ In our previous phenomenological analysis [23], we considered also suppression of this effect by a single power of $E / M_{D}$, but Eq. (11) has a better justification within our approach.

${ }^{5}$ For later reference, we recall that the above phenomenon may also be interpreted as a fluctuation in the light cone, given the refractive index mean field effect (6).
}

Born-Infeld dynamics underlying the $D$-particle recoil process [19]. Thus, in contrast to the case considered by [13], energetic particles propagate with increasingly subluminal velocities, and hence no GCR or ECR is emitted.

The non-trivial refractive index (6) has potentially observable effects on the arrival times of energetic particles from distant astrophysical objects such as gamma-ray bursters [26]. These and other suitable sources have been studied $[23,27]$, with the conclusion that $m_{Q G}>10^{15} \mathrm{GeV}$. Some tests of this retardation effect have sensitivity to larger values of $m_{Q G}$, but the corresponding bounds cannot yet be regarded as statistically established.

\section{NEUTRINO OSCILLATIONS IN NONCRITICAL STRING THEORY}

We now come to discuss a second constraint [14] on generic quantum gravity models, provided by the phenomenology of neutrino oscillations. It has been observed that, if the modifications to neutrino energy-momentum dispersion relations induced by quantum gravity are flavor dependent: $M_{Q G} \rightarrow M_{Q G_{i}}$, oscillation patterns will be affected on a distance scale $L$ of order

$$
L_{i j} \times\left(\frac{1}{M_{Q G_{i}}}-\frac{1}{M_{Q G_{j}}}\right) \sim 2 \pi \frac{1}{E^{2}}
$$

where $E$ is the typical energy. The point of [14] is that, if $M_{Q G_{i}}-M_{Q G_{j}} \sim M_{P} \sim 10^{19} \mathrm{GeV}$, the induced $L_{i j} \sim 10 \mathrm{Km}$ for $E \sim 1 \mathrm{GeV}$, which is much smaller than the characteristic oscillation length of atmospheric neutrinos, which have energies $\sim 1 \mathrm{GeV}$. It is concluded that if the $M_{Q G_{i}}$ $\sim 10^{19} \mathrm{GeV}$ they must be very nearly equal. Alternatively, the $M_{Q G_{i}} \gg 10^{19} \mathrm{GeV}$.

However, as was mentioned already in [22], in the context of a general discussion of fermion propagation through space-time foam in our noncritical Liouville string model, we expect the recoil and bubble formation effects discussed in the previous section to be essentially kinematical and geometrical, depending only on the energy of the propagating particle. As such, these phenomena should exhibit an extension of the equivalence principle, and hence be flavor independent, with no effects on oscillations. ${ }^{6}$

We recall that, since ultrahigh-energy neutrinos are not absorbed by known cosmological backgrounds, they are not subject to a GZK cutoff analogous to that expected for ultrahigh-energy protons and energetic photons. This provides the opportunity for probing particle propagation through the Universe over long distances at unequalled energies. For example, if gamma-ray bursters at cosmological distances $\sim 3000 \mathrm{Mpc}$ emit a detectable flux of neutrinos at energies up to $10^{10} \mathrm{GeV}$, as suggested in some astrophysical models, it will be possible to test quantum-gravity models with

\footnotetext{
${ }^{6} \mathrm{We}$ also recall that decoherence effects on neutrino propagation $[28,29]$ are also not expected [30] to have observable effects on present neutrino-oscillation experiments.
} 
$M_{Q G} \sim 10^{27} \mathrm{GeV}$. Alternatively, if $M_{Q G} \sim 10^{19} \mathrm{GeV}$, such ultrahigh-energy neutrino bursts would be spread out over many years, and hence unobservable [22].

\section{POSSIBLE SIGNATURES IN THE COSMIC MICROWAVE BACKGROUND RADIATION}

Another potential cosmological pitfall for quantum theories of gravity has recently been pointed out [15], namely a possible observable distortion of the cosmic microwave background (CMB) radiation spectrum. That pioneering analysis was done in the context of a model of light-cone fluctuations. Here we treat the question from the point of view of [19]. We examine here the possible effects at the source-does the thermal spectrum in a quantum-gravity model differ from that in conventional thermodynamicsand during propagation from the last scattering surface-are there observable effects of the non-trivial refractive index and stochastic effects?

The possible effect on the thermal spectrum of the CMB has to be considered from the point of view of our identification of the Liouville field with time. At finite temperatures, following the Matubara formulation of thermal field theory, we Euclideanize the Minkowski time by analytic continuation, and then compactify the time on a circle of radius $\beta$, the inverse temperature. We observe that, by construction, finitetemperature effects induce noncriticality in string theory, because the associated excitation spectrum of the finitetemperature string is in one dimension lower. Even if one started from a string theory in the critical dimension, the resulting finite-temperature string lives in a noncritical dimension. As a result, the induced central-charge deficit $Q$ always contains a term proportional to the physical temperature. In our approach of identifying time with the Liouville mode, additional departures from criticality (before compactification of the time coordinate) will also contribute to the induced central-charge deficit of the Liouville theory, and thus also to the temperature. This should be understood as a sort of back-reaction effect.

In the context of non-critical Liouville string, this process has non-trivial consequences, which we now analyze [19]. First, we remark that, in Liouville strings, the dilaton field $\Phi$ contains a term linear in the Liouville field $\phi$ :

$$
\Phi \ni \frac{1}{2} Q \phi+\cdots
$$

where $Q$ is the central-charge deficit. Therefore, when we Euclideanize the Liouville time variable $\phi$, the $\sigma$-model dilaton term, which couples to the world-sheet curvature $R^{(2)}$, becomes $^{7}$

$$
\frac{1}{2 \pi} \int_{\Sigma} \Phi R^{(2)} \rightarrow i \chi Q \phi_{E}
$$

\footnotetext{
${ }^{7} \mathrm{We}$ concentrate on the world-sheet zero mode of the Liouville field.
}

where $\chi$ is the Euler characteristic, which is $\chi=2$ for a world sheet with spherical topology. If one makes $\phi_{E}$ periodic with period $2 \pi \beta$, then, in our normalization of the Liouville terms:

$$
2 \beta Q=1
$$

i.e., the Liouville temperature is determined by the centralcharge deficit $Q$.

In non-critical string, the central-charge deficit $Q$ is in general a functional of the couplings of the various deformations of the $\sigma$ model that define the theory away from its conformal point. In the specific $D$-brane model [19], such couplings are essentially the recoil velocities $u_{D}$ of the $D$ particles in the foam. The corresponding deficit is given by $Q^{2}=c\left[u_{D}\right]-c^{*}$, where $c\left[u_{D}\right]$ is the "running central charge" of the deformed theory, and $c^{*}$ is 25 for bosonic strings or 10 for superstrings. The central-charge deficit satisfies a Zamolodchikov flow equation:

$$
\frac{\partial Q^{2}}{\partial \tau}=-\beta^{u_{D}} \mathcal{G}_{D D} \beta^{u_{D}}
$$

where $\tau$ is a renormalization-group scale on the world-sheet, $\beta^{u} D=-\left(\epsilon^{2} / 2\right) u_{D}+\mathcal{O}\left(u_{D}^{2}\right)$ is the world-sheet renormalization group $\beta$ function of the recoil operator, and $\epsilon^{-2} \sim \tau$ [19]. Since the Zamolodchikov metric $\mathcal{G}_{D D} \sim 1 / \epsilon^{2}$, and $u_{D}$ $\sim \epsilon \hat{u}$, where $\hat{u}$ is independent of the scale $\epsilon$ [19], the above flow equation may be integrated in the neighborhood of a fixed point, i.e., dropping terms of order higher than $u_{D}^{2}$, to yield the total deficit:

$$
Q \sim Q_{0} \sqrt{1+\mathcal{O}\left(u_{D}^{2}\right)}
$$

Here $Q_{0}$ a constant, associated with the equilibrium temperature in the limit of infinitely heavy $D$ particles, where recoil (back-reaction) effects are absent. The noncritical fixed point value $Q_{0} \neq 0$, because of the dimensional reduction in the spectrum of the corresponding string theory at finite temperatures that was mentioned above.

Energy-momentum conservation, which can be proven rigorously in our model [19], implies that $u_{D}^{2}$ $\sim\left(g_{s}\left(\Delta E / M_{s}\right)\right)^{2}$, where $\Delta E$ is a typical energy transfer during the scattering of a matter particle on a $D$ particle in the foam, i.e., $\Delta E=\mathcal{O}(E)$, where $E$ is the energy of the incident particle. In the case of photons with frequencies $\nu$, including the CMB photons, the above analysis implies that there will be variations in the effective temperature, related to the photon frequencies and suppressed by a power of the Planck scale $M_{s} / g_{s}$. To leading order,

$$
\delta \beta \sim \mathcal{O}\left(g_{s}^{2} \frac{\nu^{2}}{M_{s}^{2}} \beta\right)=\mathcal{O}\left(\frac{\nu^{2}}{M_{P}^{2}} \beta\right) .
$$

We consider now the effect on a thermal spectrum of frequencies $\nu$, looking for deviations from the Boltzmann form 


$$
F_{0}(\nu)=\frac{a \nu^{3}}{e^{\beta \nu}-1}
$$

where $a, \beta$ are constants. The fluctuations described above Eq. (18) cause deviations from the naive thermal spectrum of order

$$
\delta F_{0} \simeq F_{0} \frac{\nu \delta \beta e^{\beta \nu}}{e^{\beta \nu}-1}=F_{0} \frac{\nu^{3} \beta}{M_{P}^{2}} \frac{e^{\beta \nu}}{e^{\beta \nu}-1}=\mathcal{O}\left(10^{-56} F_{0}\right),
$$

for $T \sim 3000 \mathrm{~K}$ and $\nu \simeq 1 \mathrm{eV}$, typical values when the CMB was produced. This is undetectable in practice.

We now turn to the question whether effects on the propagation of $\mathrm{CMB}$ photons since their last scattering surface could have observable effects. As discussed in [23], the difference in travel times (distances travelled) of two photons with present energies $E_{1,2}$ following emission at redshift $z$ is

$$
\Delta t=\frac{\Delta L}{c}=\frac{2}{H_{0}}\left[1-\frac{1}{(1+z)^{1 / 2}}\right] \frac{E_{1}-E_{2}}{M_{Q G}} .
$$

For $E_{1}-E_{2} \sim 10^{-3} \mathrm{eV}$ and $m_{Q G} \sim 10^{19} \mathrm{GeV}$, we find $\Delta L$ $\sim 10^{-3} \mathrm{~cm}$, which is negligible compared with the thickness of the last scattering surface of the CMB. We conclude that the mean-field refractive-index effect is also undetectable in the CMB. Since the stochastic effects (11) on photon propagation are even smaller, the same conclusion applies to them, in our model.

As already commented, stochastic fluctuations in the photon velocity have effects equivalent to light-cone fluctuations. It has been suggested [15] that these might be observable in the CMB data in models [31] involving large compactified extra dimensions. We now contrast the situation in such models with the stochastic light-cone fluctuations expected in our model of Liouville quantum gravity [19], which cannot be constrained by CMB data.

We consider a thermal spectrum (19) of frequencies $\nu$, as appropriate for a CMB background. In this case, with a temperature $T=2.725 \mathrm{~K}$, the constant $\beta$ in Eq. (19) may be expressed as $\beta=1.76 \times 10^{-11}$ s. In our model, a monochromatic wave of frequency $\nu_{0}$ becomes a Gaussian with width

$$
\left.\Delta \nu\right|_{\text {Bubble }}=\left(\frac{\nu_{0}^{3}}{M_{D}^{2}}\right)
$$

each time a "bubble" is formed. Expecting these to have a characteristic size $\sim l_{P}$, the total Gaussian width we expect is

$$
\left.\Delta \nu\right|_{\text {recoil }}=\left(\frac{\nu_{0}^{3}}{M_{D}^{2}}\right) \sqrt{\frac{L}{l_{P}}} .
$$

In the model of [15], there is a characteristic light-cone fluctuation time $\Delta t$ which induces

$$
\left.\Delta \nu\right|_{\text {light-cone }}=\nu_{0}^{2} \Delta t .
$$

Using the available CMB data, it has been argued [15] that $\Delta t<2.1 \times 10^{-14}$ s. In our case, we expect that in Eq. (23) one should use $\nu_{0} \sim 10^{-3} \mathrm{eV}, M_{D} \sim 10^{19} \mathrm{GeV}, L$ $\sim 10^{10}$ ly and $l_{P} \sim 10^{-33} \mathrm{~cm}$, corresponding in the notation of [15] to $\Delta t \sim\left(\nu_{0} / M_{D}^{2}\right) \sqrt{L / l_{P}} \sim 10^{-43}$ s. This confirms our previous estimate that effects on the propagation of $\mathrm{CMB}$ photons are negligible in our noncritical Liouville-string model of space-time foam.

One may also consider stochastic (or light-cone fluctuation) effects on lines observable in the spectra of distant astrophysical objects with $z=\mathcal{O}(1)$. As in Eq. (23), we expect such spectral lines to be broadened by a fraction:

$$
\frac{\Delta \nu}{\nu} \sim\left(\frac{\nu^{2}}{M_{D}^{2}}\right) \sqrt{\frac{L}{l_{P}}} .
$$

For distances at $z \sim 1$ one has $L / l_{P} \sim 10^{61}$, and one has $\nu / M_{D} \sim 10^{-28}$ for typical atomic lines, implying $\Delta \nu / \nu$ $\sim 10^{-26}$, which is again undetectable.

\section{CONCLUSIONS}

We have explored further in the present article a model for space-time foam based on a $D$-brane world punctured by fluctuating virtual $D$-particle defects. We have explained why certain phenomena that can severely constrain other quantum-gravity models do not apply to ours.

Gravitational Cerenkov radiation is absent in our model, because energetic particles such as UHECR travel subluminally. This has been demonstrated formally, but the intuition is simply that more-energetic (higher-frequency, shorterwavelength) particles feel more strongly the iregularities (bumps) in the space-time foam.

Neutrino oscillations are absent in our model, because the effects on energetic neutrino propagation that we find are flavor independent, and so do not affect oscillation phenomenology. The basic reason for this is that the violations of four-dimensional Poincare and Lorentz invariance that we find are purely kinematic and geometric in origin.

We have estimated the effects on the CMB of expected deviations from the initial thermal spectrum, and those of our mean-field refractive-index effect and stochastic light-cone fluctuations. In each case, we find effects considerably smaller than the observational upper limits. The same is true for the spreading of spectral lines predicted in our approach.

We conclude that, although our model for space-time foam is quite radical, predicting deviations from conventional quantum mechanics and relativity that are prospectively disastrous, it escapes like Houdini from the constraints considered in this paper.

\section{ACKNOWLEDGMENTS}

N.E.M. wishes to thank H. Hofer (ETH, Zurich and CERN) for his interest and partial support. The work of D.V.N. is supported by D.O.E. grant DE-F-G03-95-ER40917. 
[1] F. David, Mod. Phys. Lett. A 3, 1651 (1988); J. Distler and H. Kawai, Nucl. Phys. B321, 509 (1989); see also N.E. Mavromatos and J.L. Miramontes, Mod. Phys. Lett. A 4, 1847 (1989); E. D'Hoker and P.S. Kurzepa, ibid. 5, 1411 (1990); E. D'Hoker, ibid. 6, 745 (1991).

[2] I. Antoniadis, C. Bachas, John Ellis, and D.V. Nanopoulos, Phys. Lett. B 211, 383 (1988); Nucl. Phys. B328, 117 (1989).

[3] J. Ellis, N.E. Mavromatos, and D.V. Nanopoulos, Phys. Lett. B 293, 37 (1992); From Supersymmetry to the Origin of SpaceTime, edited by A. Zichichi, Erice Subnuclear Series Vol. 31 (World Scientific, Singapore, 1995), p. 1, hep-th/9304133; Chaos, Solitons Fractals 10, 345 (1999).

[4] J.R. Ellis, N.E. Mavromatos, and D.V. Nanopoulos, Mod. Phys. Lett. A 12, 2813 (1997).

[5] J. Ellis, N.E. Mavromatos, and D.V. Nanopoulos, Mod. Phys. Lett. A 10, 1685 (1995).

[6] J.R. Ellis, J.S. Hagelin, D.V. Nanopoulos, and M. Srednicki, Nucl. Phys. B241, 381 (1984).

[7] J.R. Ellis, N.E. Mavromatos, and D.V. Nanopoulos, Phys. Lett. B 293, 142 (1992); P. Huet and M.E. Peskin, Nucl. Phys. B434, 3 (1995); J.R. Ellis, J.L. Lopez, N.E. Mavromatos, and D.V. Nanopoulos, Phys. Rev. D 53, 3846 (1996); F. Benatti and R. Floreanini, Nucl. Phys. B488, 335 (1997).

[8] F. Benatti, R. Floreanini, and R. Romano, Nucl. Phys. B602, 541 (2001).

[9] CPLEAR Collaboration, R. Adler et al., Phys. Lett. B 364, 239 (1995)

[10] M.A. Lawrence et al., J. Phys. G 17, 733 (1991); N.N. Efimov et al., in 22nd International Cosmic Ray Conference (1991), Dublin (Ireland); D.J. Bird et al., Astrophys. J. 441, 144 (1995); M. Takeda et al., Phys. Rev. Lett. 81, 1163 (1998); A. Watson, in Proceedings of the Snowmass Workshop (1996), p. 126; A.V. Olinto, Phys. Rep. 333-334, 329 (2000).

[11] K. Greisen, Phys. Rev. Lett. 16, 748 (1966); G.T. Zatsepin and V.A. Kuzmin, JETP Lett. 4, 78 (1966).

[12] For recent reviews, see M. Nagano, Rev. Mod. Phys. 72, 689 (2000); M. Kachelriess, astro-ph/0011231, and references therein.
[13] G.D. Moore and A. Nelson, J. High Energy Phys. 09, 023 (2001).

[14] R. Brustein, D. Eichler, and S. Foffa, hep-ph/0106309.

[15] R. Di Stefano, L.H. Ford, H. Yu, and D.J. Fixsen, astro-ph/0107001.

[16] L. Randall and R. Sundrum, Phys. Rev. Lett. 83, 4690 (1999).

[17] H. Verlinde, Nucl. Phys. B580, 264 (2000); S.S. Gubser, Phys. Rev. D 63, 084017 (2001); N. Arkani-Hamed, M. Porrati, and L. Randall, J. High Energy Phys. 08, 017 (2001); R. Ratazzi and A. Zaffaroni, ibid. 04, 021 (2001).

[18] J. Maldacena, Adv. Theor. Math. Phys. 2, 231 (1998).

[19] J. Ellis, N.E. Mavromatos, and D.V. Nanopoulos, Phys. Rev. D 61, 027503 (2000); 62, 084019 (2000); for a review, see N.E. Mavromatos, gr-qc/0009045, and references therein.

[20] J.R. Ellis, N.E. Mavromatos, and D.V. Nanopoulos, Gen. Relativ. Gravit. 32, 127 (2000).

[21] N.E. Mavromatos and R.J. Szabo, Phys. Rev. D 59, 104018 (1999).

[22] J.R. Ellis, N.E. Mavromatos, D.V. Nanopoulos, and G. Volkov, Gen. Relativ. Gravit. 32, 1777 (2000).

[23] J.R. Ellis, K. Farakos, N.E. Mavromatos, V.A. Mitsou, and D.V. Nanopoulos, Astrophys. J. 535, 139 (2000).

[24] J.R. Ellis, N.E. Mavromatos, and D.V. Nanopoulos, Phys. Rev. D 63, 124025 (2001).

[25] E. Gravanis and N.E. Mavromatos, hep-th/0103122.

[26] G. Amelino-Camelia, J. Ellis, N.E. Mavromatos, and D.V. Nanopoulos, Int. J. Mod. Phys. A 12, 607 (1997); G. AmelinoCamelia, J. Ellis, N.E. Mavromatos, D.V. Nanopoulos, and S. Sarkar, Nature (London) 393, 763 (1998).

[27] B.E. Schaefer, Phys. Rev. Lett. 82, 4964 (1999); S.D. Biller et al., ibid. 83, 2108 (1999); P. Kaaret, Astron. Astrophys. 345, L3 (1999).

[28] E. Lisi, A. Marrone, and D. Montanino, Phys. Rev. Lett. 85, 1166 (2000); F. Benatti and R. Floreanini, J. High Energy Phys. 02, 032 (2000).

[29] S.L. Adler, Phys. Rev. D 62, 117901 (2000).

[30] J.R. Ellis, N.E. Mavromatos, and D.V. Nanopoulos, Phys. Rev. D 63, 024024 (2001).

[31] H. Yu and L.H. Ford, gr-qc/0004063; Phys. Lett. B 496, 107 (2000). 